\title{
Systematic Review On Strengthening Mental Healthcare In A Fragmented Health System Through A Sustainable Financing Framework Within Counties In Kenya.
}

\author{
Milcah Ndinda Musyoki, Dr. Kezia Njoroge, Dr. Job Mapesa \\ DOI: 10.29322/IJSRP.12.01.2022.p12133 \\ http://dx.doi.org/10.29322/IJSRP.12.01.2022.p12133
}

\begin{abstract}
Financing has been highlighted as an important component that underpins the process of sustaining mental health services. This qualitative systematic review aims to identify the financial issues faced by LMIC when offering integrated primary mental health care. Using the PRISMA guide, a systematic search was carried out from relevant studies from 4 online databases, which were filtered according to inclusion and exclusion criteria. A quality assessment of the selected articles was carried out using the Critical Assessment Skills Program of the Qualitative Checklist (CASP). Using institutional theory, data was extracted on the basis of a pre-built matrix. The CERQual approach synthesized the evidence and rated the confidence level for $5 \mathrm{key}$ outcomes as low, moderate, or high. Of the 567 references identified, a total of 10 studies were included. Respondents were policy makers or implementers involved in integrated primary mental health care at the national, state, and county levels. In general, the main financial problems identified were lack of leadership and prioritization of mental health; insufficient funding and staff capacity; and negative perceptions / attitudes about mental health. The results imply that these problems must be addressed for the long-term performance of the health system. It can also improve policy formulation for better integration of primary mental health services into the health systems
\end{abstract}

\section{BACKGROUND}

A 11 Member States of W.H.O in the African region have prioritized the achievement of health and well-being as a common goal. This commitment is anchored in the Agenda for Sustainable Development as the third objective (SDG 3). This goal can only be achieved if multiple health and related goals are achieved across the SDGs. These multiple goals are grouped into three interrelated issues on which to achieve results: Universal Health Care (UHC),

Health Security (HSE), and Health Drivers Coverage (CHD). Multiple interventions are made available for populations in the region, ranging across functions of health promotion, disease prevention, curative, rehabilitative and palliative care through the life course. In practice, these compete for time and resources with the result being the interventions provided in practice are not necessarily those with the largest impact on health goals. This review is concerned with mental health.
There is a large gap in the treatment of mental disorders in lowand middle-income countries, and $70 \%$ of African countries contribute less than $1 \%$ of their health spending to mental health (World Health Organization, 2005). In the context of the growing burden of mental illness the call for an expansion of mental health services in low- and middle-income countries was prompted by the landmark 2007 Lancet series on global mental health. A study done in South Africa (Kleintjes et al., 2020) identified eight core concerns related to the National Health Insurance Bill, including the need to: (1) integrate mental health more effectively into the National Health Insurance Bill; (2) ensure equitable access to health services for all people; (3) improve human resources for mental health; (4) clarify accreditation standards and contractual conditions for service providers; (5) improve utilization of private practitioners within an integrated health system; (6) include mental health expertise in the advisory structures of the National Health Insurance Bill; (7) set out a robust accountability framework within the National Health Insurance Bill; and (8) include mental health indicators in the National Health Insurance information system.

Mental health is a human right to which every person is entitled. Every individual have the right to high quality mental health services when mental health care is needed. However, there has been a rise in mental disorders worldwide, as well as in the challenges and barriers in provision of accessible mental health services. The right to the highest attainable standard of physical and mental health remains a distant goal for millions of people. On a global level, the care available in mental health services is of poor quality, which hinders the recovery of patients and scarcity of resources is evident in many counties (Lantta et al., 2021) The term "mental health conditions" refers to a diverse spectrum of disorders including anxiety, depression, substance use disorders and schizophrenia. These conditions vary in their severity, duration and prevalence in the general population. Internationally, mental health care have mixed funding sources or limited insurance coverage and there is often a clear distinction and resulting lack of integration between psychiatric and other forms of medical care (Khoo et al., 2019)

The long-term nature of the mental illness and disability causes financial instability and disruption of family leisure, activities and family interactions. Additional cost, such as health 
care services cost, transport, assistive and personal assistance, for achieving a minimum standard of living are affected when persons with mental disabilities or their caregivers remain unemployed or under employed due to the illness/caretaking process (Nair et al., 2017)

Mental health services can be classified into three main types: community-based services, services integrated into primary care and specialized institutional or hospital services. Individual countries may face different challenges depending on type of mental health services provided. In many developing countries, restricted human resources, inaccessibility and violation of basic human right. Besides stigma, including low ranking as a health system priority, inadequate capacity in management, insufficient training of general practitioner, the absence of orientation to integrated care, high turnover of staff, inefficient intersector coordination, poor infrastructure and insufficient budget allocation (Lin et al., 2020). In developed countries for example Finland, mental health services are low-cost or free for users due to the budgeting system. However, there has been questions of inefficient use of generous resources regarding productivity of mental health services when regions in Finland and Spain are compared (Lantta et al., 2021)

Based on data from 2010 health economist estimated that mental disorders costed approximately $\$ 2.5$ trillion globally and are projected to increase to $\$ 6$ trillion by 2030 . Half of all mental illnesses occur by the age of 14 , and early childhood exposure to trauma is likely to increase a range of mental health concerns during adolescence and early adulthood (Burse et al., 2021). Globally, mental health is generally unfunded. Kenya is among the $28 \%$ of WHO member states that lack a separate budget for mental health. The global median mental health expenditure, per capita is US\$2.5 ((KHS.250). Based on the WHO Global Health Expenditure, the global average of domestic general government health expenditure per capita in 2015 was US\$ 141 (KSH 14.100), thus making government's mental health hospitals expenditure less than $2 \%$ of global average (government) health expenditure. According to office of Auditor General, Dec.2017, Kenyan government expenditure on mental health is $0.01 \%$ of the total expenditure on health. Yet, mental health conditions pose several service and financial access challenges the households. Kenyan health financing falls under four categories; out of pocket, government budgetary allocation, NHIF and private insurance. With the devolved systems of government (2013) the provision and management, of healthcare in Kenya falls under both the national government and county government. Under this system, an analysis of public health financing must be done at both national and county levels. In 2010/11, health, which was then entirely a national function, accounted for about $5.9 \%$ of total government budget, increasing in 2011/12, but it has since remained stagnant between $7-8 \%$ which is below the $15 \%$ target set by African heads of states in Abuja in 2000, of which Kenya is a signatory( Kenya Health Financing System Assessment, 2018).

The impact of burden of mental illness, unlike other illnesses, does not lie solely in the number of persons who die from the disease but mainly from the numbers of years lived with the disability following onset of the mental illness. The financial impact is substantive. Expenses for the treatment of mental illness often are borne by the family either because insurances is unavailable or because mental disorders are not fully covered by insurance ( Mental Health And Wellbeing : Towards Happiness AND National Prosperity: A report by the taskforce on Mental Health In Kenya; MOH 2020).

This paper advocates for an integrated system providing medical and behavioral care is cost-effective, as well as economically and socially desirable. An efficient and timely referral to mental health treatment is crucial to improve quality of life (DelaCruz et al., 2021). Successful attainment of UHC is a unifying goal for health system strengthening, as well as clear target for the principle of not leaving anyone behind. The concept of UHC encompasses two major areas: service coverage and financial coverage. With respect to service coverage for mental disorder, the treatment gap for low and middle-income countries is between 76 and $85 \%$. However, this huge treatment gap is not entirely due to lack of unavailability of services alone, but is also partly of financial means to pay for such services (poor financial coverage).

Successful mental health system strengthening that delivers UHC is impossible without guaranteeing financing support in a sustainable manner. Which can be identified through addressing a number of relate health system financing issues, including the sufficiency of resources for mental health, fairness in financial contributions to the costs of care, and the financial and economic impact of improved access to services. The estimates of the resources needs and the costs and health impact of scaled-up mental health service delivery.

\section{METHODS}

A systematic qualitative synthesis was carried out to obtain a comprehensive overview of existing studies on mental health care financing. This type of review summarizes published studies in a particular research area and focuses on specific research questions in a systematic and repeatable manner. Although this approach has traditionally been used to examine the effectiveness of health interventions, it has been used to develop different methods to help address a broader range of problems (for example, policy development, adequacy feasibility, and significance) related to health programs. This review was conducted by 3 public health researchers with varying levels of management experience in health, health policies and health planning. . In addition, all authors participated in face-to-face meetings and online discussions during the data collection, review, and writing phases. In general, this review consists of 5 steps: Step 1: Create a framework guide. Step 2: Conduct a systematic search and select potential items. Step 3: Carry out a critical evaluation of the selected items. Step 4: Perform data extraction and analyze the selected items. Step 5: Assess Confidence in Results.

\section{RESULT AND DISCUSSIONS}

From a study on quality of mental health services and rights of people receiving treatment in inpatient services in Finland: a 
cross- sectional observation survey with the WHO Quality Rights Tool Kit 2021; by Tella Lantta, Minna Anttila \$ Maritta Valimaki. International Journal of Mental Health Systems, Article number: 70 (2021). Where the aim of the study was to review the quality of mental services and the rights of the people receiving treatment in inpatient hospital care in Finland using the World Health Organization's QualityRight Tool Kit as a part of a randomized controlled trial VIOLIN. The results here are presented under 3 themes of perceived constrains, feasible recommendations for the attainment of increased, sustainable public and mental health financing as well as key elements for success. The findings showed that perceived constraints to increased public and mental health financing: there was low priority on mental health, this was identified as major constraint as it directly impacts on resource allocation during budgetary planning. The study found that priorities were frequently donor-driven, rather than determined on the strength of available research evidence. Lastly, fiscal pressure due to the economic recession and fluctuation in the international prices of oil had resulted in reduced government revenue and this was also identified as a constraint to increasing government funding for public, as well as mental health.

Feasible opportunities and recommended strategies for sustainable increase in public health as well as mental health financing were intiated in the country where a draft national strategic health development plan (NSHDP), included mental health services was created. This was supposed to translate into the assurance of a dedicated funding envelope for mental health services, improved political will to designate mental health as an area of government priority. This strategy was envisioned to create the opportunity to attract counterpart funding from donor agencies- including loans from the World Bank. This advocacy could be championed by technocrats, service user groups and professional association, to create more awareness for mental health; with the legislation and policy makers as the target audience.

An opportunity to link mental health with other health conditions that are currently being funded was also identified through outlining the salient mental health aspects that should be taken into consideration and implemented. E.g. perinatal depression could be tied to maternal and child health budget; or depression inked with chronic diseases, such as within the context of HIV/AIDs funding. Integrating mental health into primary care, is a strategy that can potentially result in an indirect increase in public health funding for mental health. If primary care is better funded and mental health is effectively implemented within the primary care system, services will naturally be scaled up, due to the increased resources available for primary health care. Ultimately this will result in a reduction of the treatment gap for mental disorders. Improved taxation such on every mobile phone airtime recharge, and 'sin tax' such a token on alcoholic drinks spread across the country's population can potentially generate huge resources that may be deployed for improved public health financing including mental health. These were some of the key strategies identified that could support our country strengthen mental health financing.
The second paper showed that Finnish mental health services were partially of fully achieving the standard set by the WHO QualityRights Tool Kit ( with a final score: 2.5-2.9 out of 3). The highest final score out the five theme (2.9/3) was achieved under freedom from torture or cruel, inhuman or degrading treatment or punishment and from exploitation, violence and abuse. The lowest final score out of the five themes $(2.5 / 3)$ was achieved under the right to exercise legal capacity and the right to person liberty and the security of person. Therefore, According to the findings, Finnish mental health services appear to be of high quality. However, some gaps in quality were identified, which were addressed in a national randomized controlled trial VIOLIN. Improvements could however be realized through shared decision making and relaying information to service users

The third paper on (Utilisation patterns of privately funded mental health services in Australia; Joanna Khoo, 2018) presented patient -level utilization patterns of hospital-based mental health services funded by private health insurers and examined the implications of the findings for planning and delivering private mental health services in Australia. From analyzing private health insurance claims data, this study compared differences in demographic and hospital utilization characteristics of 3,209 patients from 13 private health insurance funds with claims for other types of mental health- related hospitalization and 233,01 patients with claims for other types of hospitalizations for the period May 2014 to April 2016. Average number of overnight admissions, length of stay and per patient insurer cost are present each group. Along with overnight admissions versus same-day visits and repeat services within a 28-day period following hospitalization. Challenges in analyzing and interpreting insurance claims data to better understand private mental health service utilization are discussed. The findings indicated that patients with claims for mental health- hospitalizations were more likely to be female $62.0 \%$ compared to $55.8 \%$ male, and are significantly younger than patients with claims for other types of hospitalizations $(32.6 \%$ of patients aged 55 years and over compared to $57.1 \%$ ). Patients with claims for mental health hospitalizations have significantly higher levels of service utilization than the group with claims for types of hospitalization with a mean length of stay per overnight admission of 15.0 days ( $\mathrm{SD}=14.1$ ), a mean of 1,3 overnight admissions annually $(\mathrm{SD}=1.2)$ and mean hospital costs paid by the insurer of $\$ 13,192$ per patient ( $\mathrm{SD}=13,457)$ compared to 4.6 days $(\mathrm{SD}=7.3), 0.8$ admissions $(\mathrm{SD}=0=6)$ and $\$ 2,065$ per patient $(\mathrm{SD}=4,346)$ respectively for patients with claims for other types of hospitalizations. More than half of patients with claims for mental health-related hospitalization only claim for overnight admissions. However, the finding were difficult to interpret due to the limited information collected in insurance claims data.

The fourth paper looking at the Need for micro-finance selfgroups among women family care givers of persons with mental disability in rural India, Sreekanth Nair 2017). Proved that Microfinance self-help groups empower caregivers to indulge in productive activities based on the local availability of resources to reduce their financial burden. The purpose the paper was to assess the need for and feasibility of initiating micro-finance groups for the caregivers of persons with mental disability in rural socio- 
economical backward community of Karnataka, India. The study was a cross-sectional survey, with mixed methodology design out of the eight localities where the Mental Health Public Centres (PHC) were running successfully in Konandur area, Thirthahalli Taluk, Karnataka, one PHC was selected using simple Random Sampling Design and a $5 \mathrm{kms}$ radius from Konandur town was selected as the area of the survey (190 households). During doorto- door survey, if the family indicated that a particular member is mentally unwell, the GHQ-5 and Symptoms and Others checklist were administered on him/her and the women caregiver was interviewed using qualitative needs assessment schedule and Perceived Social Support Scale. The study findings showed there is at least a person with mental illness (5.26 per cent) in the identified households in the community. The studied proved that financial needs, capacity of the caregiver, community resources, needs, social support, burn out and stigma elicited in the interview were depicted to be the highest in and there was more need to understand the inter-connectedness between the various themes.

The fifth paper which was looking at the challenges faced by service providers on satellite mental health-care facilities in Yangon, Myanmar: a qualitative study. By Kyaw Lin Health Science, STI Myanmar University, Yangon, Myanmar Sun Lin: Mental Health Unit, Nay Pyi Taw, Myanmar and University of Public Health, Yangon, Myanmar). Aimed to assess the challenges perceived by providers in the service delivery of satellite mental healthcare (SMHC) in two sample townships in Yangon. The research was based on a case study design by applying a qualitative approach using in-depth interviews (IDIs). In the three types of service providers, a total of six staff participated as interviewees. These consisted of two team leaders, two clinical specialists providing consultations to clients and two mental health nurses. The findings indicated that providers perceived the following as major challenges in the provision of services: unstable financial resources, the lack of a functional referral system, overcrowding, inadequate individual consultation time, long-waiting hour, finites opening days and hours and poor setting of infrastructure, resulting in lack of privacy.

The sixth paper on Minister's Perceptions of Mental Health Services in Faith -Based Communities. Jacqueline R. Burse, at el. 2021). Where the purpose of this study was to explore faith-based leaders' perceptions regarding of mental health services among their congregants and within the African American Community. The study was a presentation of baseline description statistics of program evaluation data. The program being evaluation was the African American Faith -Based Mental Health Education and Awareness Initiative. The sample consisted of 264 participants, 65 male and 181 females, with $73 \%$ being 50 years and older. African Americans made up $95 \%$ of the group. Over half of the sample $(61.3 \%)$ has a bachelor' master has or doctors degree. The study findings showed that, 100 percent $(n=244)$ of pastors and clergy agree that there is a need for mental health services for members in their congregation. Also, 52.24\% $(n=128)$ disagree that they would prefer to counsel their members rather than refer them to a mental health professional. According to another study from South Africa shows that there are multitude of factors that have weakened the provision of mental health services in South Africa, mostly critically the lack of human and financial resources to address treatment gaps. Limited routine information systems to understand the true burden of mental disorders and utilization patterns and high levels of stigma. As a result of poor access to good access to good quality primary mental health care, the entry level for accessing mental health services at present is mostly at an inappropriate level care. This is significantly contributed to high costs of health care and inefficiency of health system. This has also meant that care-seeking typically occurs when patients experience very severe symptoms, largely as a result of untreated mental illness and often require long-term institutionalized without much potential of returning to their communities. This is largely due to limited availability of well-resourced communitybased residential and day care services to manage mental health care users after discharge, coupled with impact of poverty on households with families unable or unwilling to care for family members after discharge. The National Government of South Africa uses two types of transfers, conditional grants and unconditional provincial equitable share funds, to send money to provinces in South Africa. Presently South Africa does not have a ring-fenced budget for mental health and funding falls under general health allocations of the equitable share. Further, the absence of ring-fenced allocations for the development and maintenance of the specialized psychiatric service at the tertiary level has created a number of substantial challenges: the psychiatric hospitals are out dated and in disrepair, there is acute shortage of mental health professionals available to deliver this service, the facilities are unable to invest in the advancement of their scope of service. The mental health NGOs in South Africa are struggling with sustainability at present. To secure funding from the Department of Social Development and health most mental health NGOs in South Africa have needed to commit to the delivery of statuary interventions, not mental health services. The stake holders believe that due to these models of funding, their mental health services become diluted and unspecialized focusing on family planning, or foster care or services to the aged (Docrat et al., 2019)

The seventh paper on Cost - Effectiveness Analysis Combining Medical and Mental Health Servises for Older Adults with HIV in New York City by Juan J. Delacruz, Christos Giannikos . Andreas Kakolyris R). To understand the differential in cost and effectiveness of an additional unit of perfect health. The study evaluated two rival health interventions using a standard decision-analytic model and quantified the cost per qualityadjusted life-year (QALY) to understand the differential in cost and effectiveness of an additional unit of perfect health. HIV medical and behavioral care. Primary data from a convenience sample $(n=139)$ collected in New York City and outcomes for healthy older adults from the literature were used in the study. The results showed that the incremental cost-effectiveness ratio $\$ 36,166$ per QALY, which is less than the willingness to pay $(\$ 75,000)$. The ICER for Hispanics was $\$ 35,325$ and for Whites/Caucasians was $\$ 40,499$. Integrated medical plus behaviour care is cost-effective and improves quality of life among OAWH.

The eighth paper on Donor Financing of Global Mental Health, 1995-2015: An Assessment of Trends, Channels and Alignment with thw Disease Burden : By F.J Charison, J. 
Dieleman, L., Singn, H.A. Whiteford, 2017). Where the paper extended previous assessment of development assistance for mental health (DAMH) in two significant ways; first by contrasting DAMH against that that for other disease categories and second by benchmarking allocated development assistance against the core disease the core disease burden metric ( disability - adjusted life year) as estimated by the Global Burden of Disease Studies. In order to track DAH, IHME collates information from audited financial recorders, project level data, and budget information from the primary global health channels. The diverse set of data were standardized and put into a single inflation adjusted current ( 2015 US dollars and each dollar disbursed was assigned up to one health focus areas from 1990 through 2015. The study tied those health financing estimates to disease burden estimates (DALYs) produced by the Global Burden of Disease 2015 Study to calculated a standardized measure across health focus area-development assistance for health (in US Dollars) per DALY. The study findings proved that DAMH increased from USD 18million in 1995 to usd 132 million in 2015, which equates to $0.4 \%$ of total DAH IN 2015, private philanthropy was the most significant source (USD 435 million, $30 \%$ of DAMH), while the United States government provided USD 270 million of total DAMH. South and Southeast Asia received the largest proportion of funding for mental health in 2013 (34\%), DAMH available per DALY in 2013 ranged from USD 0.27 in East Asia and the Pacific to USD 1.8 in the Middle East and North Africa. HIV/AIDs received the largest ratio of funds to burden - approximately USD 145 Per DALY in 2013. Mental and substance use disorders and its broader category of non-communicable disease received less than USD1 of DAH per DALY.

The ninth paper a study in Nigeria showed public health insurance remains the most feasible and sustainable financing intervention for scaling up mental health care services. It is the only health financing form that possesses the important characteristics of universality, price regulation, open enrolment, and a defined and regulated benefits package expected of any dynamic health financing means. (Abdulmalik et al., 2019)

Fig. 1 Proposed model of mental health financing for Nigeria

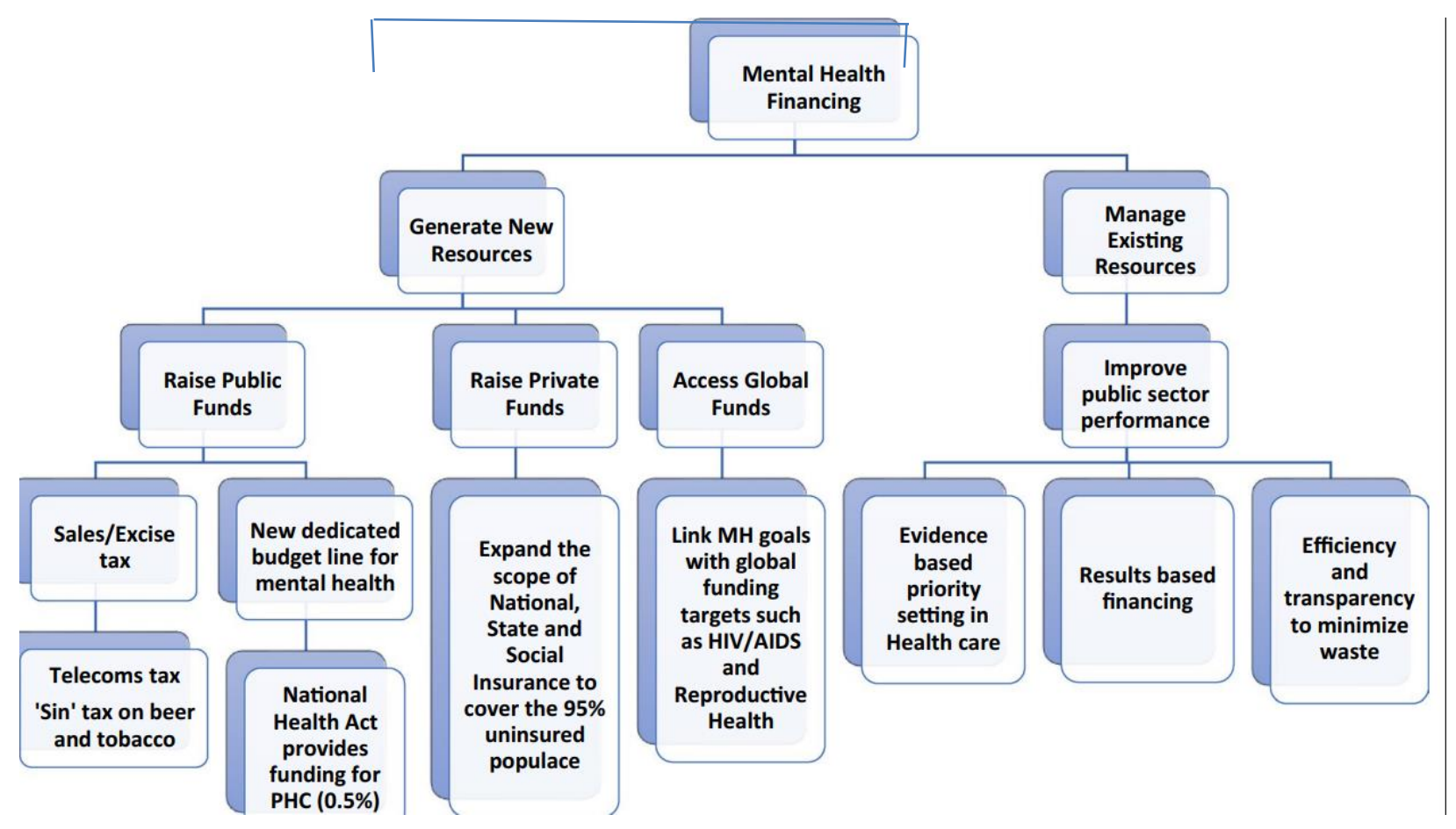

The tenth paper a study in Chicago show that lack of public funding for mental health services is compounded by the fact that few private foundations fund in mental health and those that do have not focused on the links between trauma and mental health (Warshaw et al., 2003). According to F.J Charison, 2017, apparent inequities extended well beyond the total proportion of development assistance allocated to mental health. Private philanthropy accounts for only a fraction of total DAH yet it is overwhelming the largest donor of DAMH suggesting a lack of interest by governments to address mental health needs across the globe. In terms of recipients, there appears to be no clear association between need, in terms of absolute DALYs and where DAMH is going. There was also other important indictors which highlight the lack of resources in mental health was that according to the World Health Organization Mental Atlas, there is one psychiatrist per 200,000 people or more for half of the world's population. Around $80 \%$ of mental healthcare workers are based in inpatient and day care services. The capacity to build the workforce appears minimal with the same report estimating around $2 \%$ of physicians and nurses received at least 2 days of 
mental health training in the 2 years. Furthermore, funding for research into mental illness is not on par with research funding allocated to physical conditions. Even in the face of economic arguments, as well as burden of disease evidence, governments in low and middle income countries have been slow to respond to the rising burden of mental and substance use disorders. The well known study undertaken for World Economic Forum estimated that the cumulative global impact of mental disorders terms of lost economic output may amount to US\$16 trillion over 20 years, equivalent to $25 \%$ of global GDP in 2010 . WHO found that a scaled -up package of mental health intervention for mental disorders in Sub-Saharan-African and South Asia, would cost in the order of US\$3-4 per head of population.

Using criteria such as these, an analysis for the World Bank found a strong case for government and public sector involvement in mental health treatment. Mental health has not, in most countries, become a priority commensurate with the extent of its burden and the potential to reduce the burden. According to World Economic Forum, mental illness is still perceived as an individual or family problem rather than policy challenge with significant economic and political implications, and in many low and middleincome countries, treatment for mental illness is seen as an unaffordable luxury. Tackling perceptions such as these will require a more sophisticated presentation of evidence to governments, funders and society than has been achieved to date. It is hoped that actions such as the inclusion of mental health in sustainable Development Goals and commitments from major stakeholders in global health, such as those given at the April 2016 meeting, co-hosted by the World Bank and WHO, to make mental health a global health and development priority will coalesce with mental health campaigns and movements within and across societies, to create the tipping point for mental health to last become a global health priority (Charlson et al., 2017)

\section{CONCLUSION}

It is evident that the burden of mental health is on the increase in both developed and developing countries. Some of the causes of mental health include torture, cruelty, abuse and stress. Most of the mentally affected people are women. There are multitude of factors that have weakened the provision of mental health services in most health systems, mostly lack of human and financial resources, limited routine information systems, and low priority on mental health issues.

The quality of mental health services is compromised due to perceived constrains of resources. Therefore leading to low priority on mental health issues. Hence there is need to improve resource allocation during budgetary planning. There is also need more advocacy for mental health issues to create awareness for mental health among policy makers.

There is need to ensure financial needs for mental illinesses are met, building capacity of caregivers, social support for caregivers to reduce burnouts and stigma. The lack of governments to support mental health affects donors funding as well.

Even where the burden I high and cost-effective treatment exist, other factors influence government and funders. They include the importance of metal health as Public good, the societal impact of untreated mental illness (externalities), the need for regulation (including of service providers), protection from catastrophic costs and whether the private sector can provide mental health services.

\section{REFERENCES}

[1] Abdulmalik, J., Olayiwola, S., Docrat, S., Lund, C., Chisholm, D., \& Gureje O. (2019). Sustainable financing mechanisms for strengthening mental health systems in Nigeria. International Journal of Mental Health Systems, 13(1), 38. https://doi.org/10.1186/s13033-019-0293-8

[2] Burse, J., Barnett, T. M., Collins, L. R., Osborne, A. S., \& Stewart, N. (2021) Ministers' Perceptions of Faith-Based Communities in Mental Health Services. Social Work \& Christianity, 48(2), 137-155. https://doi.org/10.34043/swc.v48i2.101

[3] Charlson, F. J., Dieleman, J., Singh, L., \& Whiteford, H. A. (2017). Donor Financing of Global Mental Health, 1995-2015: An Assessment of Trends, Channels, and Alignment with the Disease Burden. PloS One, 12(1), e0169384. https://doi.org/10.1371/journal.pone.0169384

[4] DelaCruz, J. J., Giannikos, C., Kakolyris, A., Utzinger, R. C., \& Karpiak, S E. (2021). Cost-Effectiveness Analysis Combining Medical and Mental Health Services for Older Adults with HIV in New York City. Atlantic Economic Journal: AEJ, 49(1), 43-56. https://doi.org/10.1007/s11293-02109697-3

[5] Docrat, S., Lund, C., \& Chisholm, D. (2019). Sustainable financing options for mental health care in South Africa: Findings from a situation analysis and key informant interviews. International Journal of Mental Health Systems, 13(1), 4. https://doi.org/10.1186/s13033-019-0260-4

[6] Khoo, J., Hasan, H., \& Eagar, K. (2019). Utilisation patterns of privately funded mental health services in Australia. Journal of Health Organization and Management, 33(1), 5-17. https://doi.org/10.1108/JHOM-02-2018-0062

[7] Kleintjes, S., Hollander, D. H. den, Pillay, S. R., \& Kramers-Olen, A. (2020). Strengthening the National Health Insurance Bill for mental health needs: Response from the Psychological Society of South Africa: South African Journal of Psychology. https://doi.org/10.1177/0081246320954317

[8] Lantta, T., Anttila, M., \& Välimäki, M. (2021). Quality of mental health services and rights of people receiving treatment in inpatient services in Finland: A cross-sectional observational survey with the WHO QualityRights Tool Kit. International Journal of Mental Health Systems, 15(1), 70. https://doi.org/10.1186/s13033-021-00495-7

[9] Lin, K., Lin, S., \& Sein, T. T. (2020). The challenges faced by service providers on satellite mental health-care facilities in Yangon, Myanmar: A qualitative study. Journal of Health Research, ahead-of-print(ahead-of-print). https://doi.org/10.1108/JHR-02-2020-0029

[10] Nair, S. S., Jagannathan, A., Kudumallige, S., Kumar, C., \& Thirthalli, J. (2017). Need for micro-finance self-help groups among women family caregivers of persons with mental disability in rural India. https://doi.org/10.1108/MHSI-10-2017-0039

[11] Warshaw, C., Gugenheim, A. M., Moroney, G., \& Barnes, H. (2003) Fragmented Services, Unmet Needs: Building Collaboration Between The Mental Health And Domestic Violence Communities. Health Affairs, 22(5), 230-234. https://doi.org/10.1377/hlthaff.22.5.230

\section{AUTHORS}

First Author - Milcah Ndinda Musyoki

Second Author - Dr. Kezia Njoroge

Third Author - Dr. Job Mapesa 
International Journal of Scientific and Research Publications, Volume 12, Issue 1, January 2022

This publication is licensed under Creative Commons Attribution CC BY. 